\title{
Flora of spoil heaps after hard coal mining in Trzebinia (southern Poland): effect of substratum properties
}

\author{
Marcin W. Woch ${ }^{1}{ }^{*}$, Magdalena Radwańska ${ }^{1}$, Anna M. Stefanowicz ${ }^{2}$ \\ ${ }^{1}$ Department of Botany, Institute of Biology, Pedagogical University of Kraków, \\ Podchorążych 2, Kraków 31-084, Poland \\ ${ }^{2}$ Department of Ecology, Institute of Botany, Polish Academy of Sciences, Lubicz 46, \\ 31-512 Kraków, Poland
}

\begin{abstract}
The aim of the present study was to investigate the composition of spontaneous plant cover and the physicochemical properties of the substratum of spoil heaps of the Siersza hard coal mine in Trzebinia (southern Poland) abandoned in 2001. Floristic and soil analyses were performed in 2011. The substratum was very diverse in terms of texture (sand: 55-92 \%, clay: 6-38\%), nutrient content (total C: $1.3-41.0 \%$, total N: $0.05-0.49 \%$, total Ca: $0.5-7.3 \%)$ and $\mathrm{pH}(3.7-8.7)$. Moreover, total thallium concentration in the substratum was high, ranging from 6.0 to $14.6 \mathrm{mg} \mathrm{kg}^{-1}$. Plant cover varied from 50 to $95 \%$. The number of plant species per $4 \mathrm{~m}^{2}$ varied from 6 to 29 and correlated negatively with total carbon content $(\mathrm{r}=-0.85, \mathrm{p}<0.01)$, and positively with sand content in the substratum $(\mathrm{r}=66, \mathrm{p}<0.05)$. The highest number of species per area unit was observed on a humus substratum, where initial soil has developed on the part of carboniferous waste rock spoil under 20-30 year old trees, and the lowest on carbon shale with coal and culm. Among 197 plant species, most belong to Asteraceae, Fabaceae, Poaceae and Rosaceae families. Hemicryptophytes (49\%) and terophytes (18\%) predominated. The investigated area was primarily colonized by native species spread by the wind. However, invasive alien species also had a significant share $(8 \%)$ in the plant cover.
\end{abstract}

Key words: carbon, coal spoil heap, seed dispersion, species richness, succession, thallium, Trzebinia, Poland

\section{Introduction}

The increasing number of post-industrial wastelands are resulting in considerable changes in the landscape and vegetation cover. The large hard coal deposits and the fact that coal is the main energy raw material in Poland have encouraged mining with its related spoil storage. Heaps of waste rock have become a frequent part of the landscape in southern Poland, especially in the Upper Silesia coalfield as well as in the smaller Kraków coalfield

\footnotetext{
* Corresponding author, e-mail: jurania@o2.pl

Copyright $^{\circledR} 2013$ by Acta Botanica Croatica, the Faculty of Science, University of Zagreb. All rights reserved.
} 
adjoining it from the east. The pioneer flora and vegetation of the hard coal mining waste dumps have been the subject of numerous papers (e.g. CABAŁA and SYPIEŃ 1987; TOKARSKA-GuZIK et al.1991; CABAŁA and JARZĄBEK 1999; RostAŃSKI 2000, 2006; WORYNA and ROSTAŃSKI 2003; WOŹNIAK 1998, 2010). However, there is still a need for more floristic research analyzing the properties of specific substrata of these anthropogenic habitats. Previously, little botanic research has dealt with the issue of substratum characteristics (BAIG 1992, RosTAŃSKI 2006, WoŹNIAK 2010, MAKINECI et al. 2011). The relatively wide variety of species of post-mining dumping grounds includes both native species as well as those alien to Central European flora, which have ecological amplitudes enabling them to colonize such places. It is especially vital to discover the edaphic reasons for the differentiation of the flora growing on the post-mining dumping grounds, and whether the changes in the substratum in time or its spatial heterogeneity are more decisive (GIBSON et al. 1985, PiCKET et al.1987, LUKEN 1990, Holl and CAIRns 2002, WoźNIAK 2010). The aim of the research presented in this paper was to study the species composition of the plants growing on the post-mining spoil heaps and wastelands of the disused Siersza mine (KWK Siersza) in Trzebinia, where spontaneous plant succession (colonization and change in the community structure over time) have been taking place (KREBS 2009). The most important types of substratum occurring in the studied area were classified, for which analyses of physicochemical properties as basic factors determining the flora makeup were performed.

\section{Material and methods}

\section{Study site}

The wastelands of the Siersza hard coal mine (KWK Siersza) studied lie in Trzebinia in north-western Malopolska (50 $\left.19^{\prime} 072^{\prime \prime} \mathrm{N}, 20^{\circ} 44^{\prime} 6938^{\prime \prime} \mathrm{E}\right)$. The beginnings of this mine date back to the year 1808 and it operated until 1999 (KIRYK 1994). In 2001, the post-mining waste left behind on the surface was condensed with a vibrating roller, the upper layer being left loose in order to facilitate plant colonization. The area was leveled off, the slope was moderated in the direction of the rail tracks and soil was distributed in some parts. The research area was constituted by post-mining waste dumps as well as wastelands remaining after the buildings had been demolished; altogether the site covers an area of about 20 ha. The remaining habitats studied were also illegal rubbish dumps, areas storing debris from demolished administrative buildings as well singular ash mounds from the Siersza Power Plant. The studied area is located in the Śląsk-Kraków Highlands (Pagóry Myślachowickie), located at $340 \mathrm{~m}$ above sea level (KONDRACKI 2000). Post-mining wastelands are mostly surrounded by forest areas - directly from the north, while in the south the forests are situated behind a local railway line delivering coal to the nearby Siersza Power Plant and a street. In the west and east, the area studied borders with the low-density residential areas of the Siersza and Kopalnia estates (Fig.1).

\section{Climate and vegetation}

The Kraków coalfield is in a transitional climate zone, between a moderate oceanic climate in the west and a moderate continental climate in the east. Various air masses collide in this area, which is a result of the location in the centre of Europe as well as a latitudinal system of geographical lands. The average annual air temperature is $7.8^{\circ} \mathrm{C}$, while the annual rainfall $770-811 \mathrm{~mm}$. The vegetation season spans between 205 and 215 days. In 


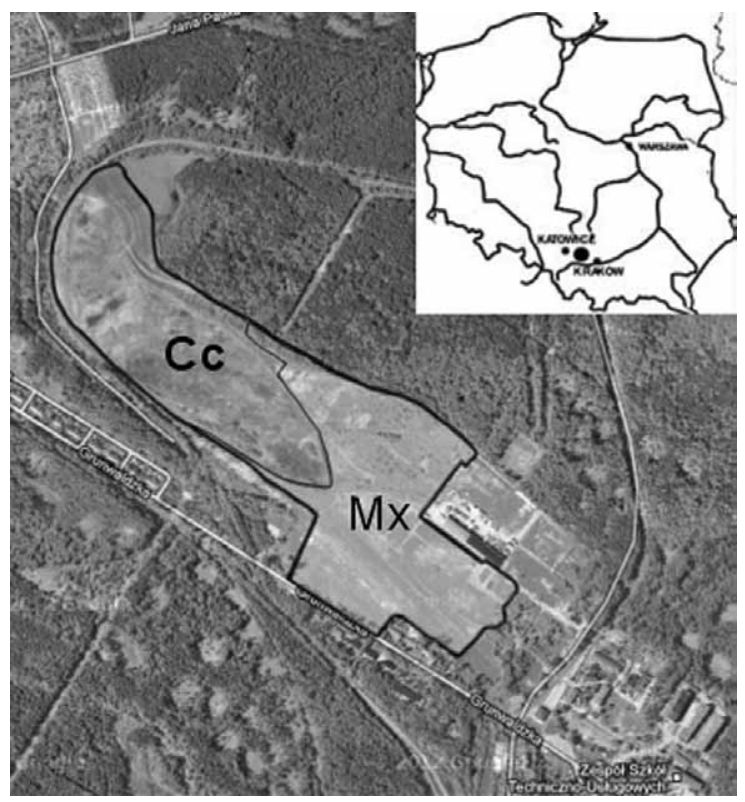

Fig. 1. Study site with two dominating types of substratum: $\mathbf{C c}$-carboniferous shale with coal, Mx - mixed substratum. Retrieved in 2012 from GoogleEarth and modified.

this area, westerly winds are the most frequent, followed by easterly winds (WITKOWSKA-KITA et al. 2010). Trzebinia has been traditionally strongly connected with industry, therefore its plant cover has been largely transformed. In the flora of Trzebinia there are 650 plant species, 40 of which are protected in Poland (SUDER and CABAŁA 2004; Woch 2005, 2007, 2012). A high share of alien species (anthropophytes) - 19.8\% (SUDER and CABAŁA 2004) indicates significant synanthropization. Hemicryptophytes are the dominating life forms (39.2\%), followed by terophytes (12\%) (SUDER and CABAŁA 2004). Artificially planted pine monocultures dominate the forests; there are also impoverished beech forests as well as riparian deciduous forests. Generally, forests cover $43.6 \%$ of the municipality (WiTKOWSKA-KitA et al. 2010).

\section{Field survey}

The anthropogenic substratum occurring in the studied area can be divided into six types - two spatially dominating: (1) carboniferous shale with coal (about 12 ha), (2) mixed substratum (about $7 \mathrm{ha}$ ) and four spatially marginal (no more than $1 \mathrm{ha}$ ): (3) culm substratum; (4) humus substratum under 20-30 year old trees of Betula pendula, Pinus sylvestris, Populus tremula and Salix caprea; (5) illegal dumps of construction debris with rubbish and single mounds of (6) coal ashes from the power plant. Floristic data were compiled during the 2011 vegetation season in patches of each substratum type. To assess the number of plant species per area unit, 10 plots of $4 \mathrm{~m}^{2}$ were established in the centers of the most typical patches of each substratum type. A floristic list was drawn up at each plot. Additionally, 3 samples of substratum/soil to a depth of $15 \mathrm{~cm}$ were collected and bulked to obtain one composite sample from each plot. 


\section{Floristic analyses}

To make a complete list of plant species occurring on 20 ha area of the wastelands of the Siersza hard coal mine, the occurrence of each plant species was estimated, taking 6 substratum types into consideration. While analyzing the flora, species frequency, share of geographical and historical groups (RUTKOWSKI 2004, SUDNIK-WóJCIKOWSKA 2011), share of life forms (RUTKOWSKI 2004) as well as the seed proliferation (PODBIELKOWSKI 1995, SUDNIK-WóJCIKOWSKA 2011) were taken into account. The Latin nomenclature follows RUTKOWSKI (2004).

\section{Soil analyses}

Before analysis, soil samples were sieved ( $2 \mathrm{~mm}$ mesh). Soil texture was determined by a combination of sieving and sedimentation (INTERNATIONAL ORGANIZATION FOR STANDARDIZATION 1998). Soil $\mathrm{pH}$ was measured with a $\mathrm{pH}$-meter after extraction with $\mathrm{H}_{2} \mathrm{O}$ at a 1:5 (w:v) ratio (INTERNATIONAL ORGANIZATION FOR STANDARDIZATION 1994). Total carbon was assessed by dry combustion technique with a Leco RC-612 (INTERNATIONAL ORGANIZATION FOR STANDARDIZATION 1995a), and sulfur with a Leco SC-144 DR. Nitrogen was measured by a method based on Kjeldahl digestion (Kjeltec 2300, Foss Tecator; INTERNATIONAL ORGANiZATION For StANDARDiZATION 1995b). Total Cd, Pb, Zn, Tl, Mn and Mg in soil were measured with the use of atomic absorption spectrometry (Varian 220 FS) after digestion in hot concentrated $\mathrm{HClO}_{4}$, exchangeable $\mathrm{Cd}, \mathrm{Pb}, \mathrm{Zn}, \mathrm{Tl}, \mathrm{Mn}, \mathrm{Fe}, \mathrm{Na}, \mathrm{K}, \mathrm{Ca}, \mathrm{Mg}$ after extraction with $0.1 \mathrm{M} \mathrm{BaCl}_{2}$ (pH 7.0) (INTERNATIONAL ORGANIZATION For STANDARDIZATION 1995c, modified), and water-soluble $\mathrm{Cd}, \mathrm{Pb}$ and $\mathrm{Zn}$ after extraction with deionized water. Phosphorus was measured with the molybdenum blue method after digestion in hot $\mathrm{HClO}_{4}$ (total) or after extraction with $0.5 \mathrm{M} \mathrm{NaHCO}_{3}$ (OLSEN et al. 1954) with a colorimeter (Hach-Lange DR 3800).

\section{Results}

The study area was differentiated in terms of the physicochemical properties of the substratum (Tab. 1). For example, sand and clay content ranged from 55 to $92 \%$ and from 6 to $38 \%$, respectively. Soil $\mathrm{pH}$ varied from 3.7 to 8.7 , total C content from 1.3 to $41.0 \%$, total N from 0.05 to $0.49 \%$, total Ca from 0.5 to $7.3 \%$, and total P from 221 to $914 \mathrm{mg} \mathrm{kg}^{-1}$. Large differences between sites were also found in concentrations of available element forms. For example, exchangeable Ca ranged from 52 to $3039 \mathrm{mg} \mathrm{kg}^{-1}$, exchangeable $\mathrm{Mg}$ from 1.8 to $1132 \mathrm{mg} \mathrm{kg}^{-1}$, and available P from 0.1 to $14.8 \mathrm{mg} \mathrm{kg}^{-1}$. Total content of heavy metals such as $\mathrm{Cd}, \mathrm{Pb}$ and $\mathrm{Zn}$ in the substratum of many sites was slightly elevated, exceeding $5 \mathrm{mg} \mathrm{Cd}$ $\mathrm{kg}^{-1}, 260 \mathrm{mg} \mathrm{Pb} \mathrm{kg}^{-1}$ and $500 \mathrm{mg} \mathrm{Zn} \mathrm{kg}^{-1}$. In turn, total Tl concentration was high at all studied sites and ranged from 6.0 to $14.6 \mathrm{mg} \mathrm{kg}^{-1}$.

\section{Characteristics of main types of substratum}

\section{Carboniferous shale with coal}

The prevailing substratum type in the studied area consisted of carboniferous shale and coal. Some physicochemical properties of soil developed on this substratum were diverse. This was especially observed in the case of S, exchangeable Fe, Mn and Zn as well as water- 
Tab. 1. Physicochemical properties of substratum and vegetation parameters at studied sites. Values for $\mathrm{Pb}_{\mathrm{ex}}, \mathrm{Tl}_{\mathrm{ex}}$ and $\mathrm{Cd}_{\mathrm{ws}}$ were below detection limits in all soils, so they are not shown in the table; $\mathbf{D}$ - illegal dumps of construction debris with rubbish, $\mathbf{C c}$ - carbon shale with coal, Co - coal ashes, Hu- humus subsoil, $\mathbf{C m}$ - culm subsoil, $\mathbf{M x}$ - mixed substratum; $\mathbf{S}_{\text {plant }}$ number of plant species, $\mathbf{C o v}$ - plant cover; tot $-\mathbf{H C l O}_{4}$-extractable, $\mathbf{e x}-\mathrm{BaCl}_{2}$-extractable, ws - water soluble, av - available (Olsen method); bdl - below detection limit.

\begin{tabular}{|c|c|c|c|c|c|c|c|c|c|c|}
\hline Site & 1 & 2 & 3 & 4 & 5 & 6 & 7 & 8 & 9 & 10 \\
\hline subsoil type & $\mathrm{D}$ & $\mathrm{Cc}$ & $\mathrm{D}$ & $\mathrm{Cc}$ & $\mathrm{Cc}$ & Co & $\mathrm{Hu}$ & $\mathrm{Cm}$ & Mx & $\mathrm{Hu}$ \\
\hline Sand (\%) & 82 & 59 & 79 & 71 & 76 & 79 & 90 & 55 & 92 & 81 \\
\hline Silt (\%) & 3 & 9 & 5 & 4 & 1 & 10 & 4 & 7 & 2 & 5 \\
\hline Clay (\%) & 15 & 32 & 16 & 25 & 23 & 11 & 6 & 38 & 6 & 14 \\
\hline $\mathrm{C}_{\mathrm{tot}}(\%)$ & 1.5 & 23.9 & 2.2 & 14.5 & 11.6 & 5.9 & 5.8 & 41.0 & 1.3 & 3.3 \\
\hline $\mathrm{N}_{\text {tot }}(\%)$ & 0.49 & 0.05 & 0.07 & 0.20 & 0.28 & 0.05 & 0.17 & 0.48 & 0.12 & 0.06 \\
\hline $\mathrm{S}_{\mathrm{tot}}(\%)$ & 0.42 & 0.02 & 0.94 & 0.04 & 1.00 & 0.98 & 0.19 & 0.12 & 0.98 & 0.04 \\
\hline $\mathrm{Ca}_{\text {tot }}(\%)$ & 6.02 & 0.51 & 2.58 & 1.35 & 1.32 & 2.68 & 7.31 & 0.68 & 1.81 & 0.79 \\
\hline $\mathrm{Cd}_{\text {tot }}\left(\mathrm{mg} \mathrm{kg}^{-1}\right)$ & 3.45 & 1.45 & 5.31 & 3.15 & 5.56 & 1.59 & 2.76 & 2.97 & 1.28 & 1.83 \\
\hline $\mathrm{Fe}_{\mathrm{tot}}(\%)$ & 0.95 & 1.50 & 0.60 & 2.22 & 2.01 & 2.30 & 2.15 & 1.48 & 1.00 & 2.11 \\
\hline $\mathrm{K}_{\mathrm{tot}}(\%)$ & 0.33 & 0.57 & 0.17 & 0.39 & 0.58 & 0.26 & 0.14 & 0.49 & 0.09 & 0.25 \\
\hline $\mathrm{Mg}_{\text {tot }}(\%)$ & 0.49 & 0.27 & 0.38 & 0.41 & 0.53 & 1.04 & 0.82 & 0.49 & 0.09 & 0.20 \\
\hline $\mathrm{Mn}_{\text {tot }}\left(\mathrm{mg} \mathrm{kg}^{-1}\right)$ & 238 & 125 & 259 & 180 & 346 & 255 & 1509 & 321 & 134 & 448 \\
\hline $\mathrm{Na}_{\text {tot }}\left(\mathrm{mg} \mathrm{kg}^{-1}\right)$ & 132 & 282 & 123 & 233 & 296 & 914 & 107 & 258 & 163 & 132 \\
\hline $\mathrm{P}_{\text {tot }}\left(\mathrm{mg} \mathrm{kg}^{-1}\right)$ & 914 & 351 & 493 & 342 & 365 & 838 & 651 & 336 & 221 & 367 \\
\hline $\mathrm{Pb}_{\text {tot }}\left(\mathrm{mg} \mathrm{kg}^{-1}\right)$ & 84 & 69 & 163 & 263 & 240 & 49 & 101 & 107 & 32 & 85 \\
\hline $\mathrm{Tl}_{\text {tot }}\left(\mathrm{mg} \mathrm{kg}^{-1}\right)$ & 9.91 & 12.37 & 7.79 & 11.06 & 14.01 & 11.73 & 14.59 & 12.51 & 5.98 & 9.65 \\
\hline $\mathrm{Zn}_{\text {tot }}\left(\mathrm{mg} \mathrm{kg}^{-1}\right)$ & 415 & 137 & 510 & 417 & 456 & 207 & 272 & 343 & 84 & 169 \\
\hline $\mathrm{Ca}_{\mathrm{ex}}\left(\mathrm{mg} \mathrm{kg}^{-1}\right)$ & 1716 & 1140 & 1460 & 2792 & 2288 & 646 & 3039 & 52 & 885 & 2428 \\
\hline $\mathrm{Cd}_{\mathrm{ex}}\left(\mathrm{mg} \mathrm{kg}^{-1}\right)$ & bdl & 0.22 & 0.22 & 0.07 & 0.77 & 0.62 & 0.63 & bdl & 0.64 & 0.63 \\
\hline $\mathrm{Fe}_{\mathrm{ex}}\left(\mathrm{mg} \mathrm{kg}^{-1}\right)$ & 2.04 & 29.36 & 2.11 & 2.52 & 3.19 & 2.14 & 2.06 & 2.43 & 2.24 & 1.97 \\
\hline $\mathrm{K}_{\mathrm{ex}}\left(\mathrm{mg} \mathrm{kg}^{-1}\right)$ & 58.3 & 36.5 & 93.8 & 48.0 & 25.9 & 38.5 & 61.9 & 70.9 & 40.0 & 59.9 \\
\hline $\operatorname{Mg}_{\text {ex }}\left(\mathrm{mg} \mathrm{kg}^{-1}\right)$ & 77.8 & 330 & 62.2 & 432 & 480 & 137 & 364 & 1132 & 1.8 & 110 \\
\hline $\mathrm{Mn}_{\mathrm{ex}}\left(\mathrm{mg} \mathrm{kg}^{-1}\right)$ & bdl & 21.19 & 0.31 & 2.48 & 14.68 & bdl & 1.27 & 18.64 & bdl & 0.52 \\
\hline $\mathrm{Na}_{\mathrm{ex}}\left(\mathrm{mg} \mathrm{kg}^{-1}\right)$ & 11.1 & 16.0 & 11.9 & 22.5 & 14.4 & 11.0 & 10.3 & 27.7 & 105.9 & 10.4 \\
\hline $\mathrm{Zn}_{\mathrm{ex}}\left(\mathrm{mg} \mathrm{kg}^{-1}\right)$ & 0.36 & 40.39 & 0.73 & 2.41 & 28.53 & bdl & bdl & 8.90 & bdl & 0.18 \\
\hline $\mathrm{P}_{\mathrm{av}}\left(\mathrm{mg} \mathrm{kg}^{-1}\right)$ & 6.01 & 13.32 & 10.52 & 2.89 & 14.77 & 6.83 & 2.64 & 0.07 & 0.08 & 0.18 \\
\hline $\mathrm{Zn}_{\mathrm{ws}}\left(\mathrm{mg} \mathrm{kg}^{-1}\right)$ & 0.18 & 22.71 & 2.27 & 0.64 & 4.11 & 1.42 & 0.16 & 0.54 & 0.21 & 0.19 \\
\hline $\mathrm{Pb}_{\mathrm{ws}}\left(\mu \mathrm{g} \mathrm{kg}^{-1}\right)$ & 484 & 290 & 883 & 353 & 257 & 822 & 365 & 388 & 412 & 239 \\
\hline $\mathrm{pH}\left(\mathrm{H}_{2} \mathrm{O}\right)$ & 8.4 & 3.7 & 8.2 & 6.3 & 5.8 & 8.2 & 7.7 & 6.0 & 8.7 & 7.4 \\
\hline$S_{\text {plant }}^{\prime}$ & 23 & 7 & 24 & 8 & 20 & 20 & 18 & 6 & 23 & 29 \\
\hline $\operatorname{Cov}(\%)$ & 50 & 90 & 60 & 80 & 95 & 75 & 65 & 80 & 85 & 60 \\
\hline
\end{tabular}


-soluble $\mathrm{Zn}$ and $\mathrm{pH}$. The soil was an acidic sandy clay loam, characterized by high carbon content. Total K, Pb and $\mathrm{Zn}$ as well as exchangeable Fe, Mn, Zn, available P and water-soluble $\mathrm{Zn}$ contents in soil were high in comparison to other soils. These sites were dominated by Agrostis stolonifera, Calamagrostis epigejos and Tanacetum vulgare (Tab. 1, sites 2, 4 and 5).

\section{Mixed substratum}

A mixed substratum was the second dominant substratum type. It was a mixture of carboniferous shale and coal, debris originating from demolished mine buildings, brought-in soil, sand and dolomite stones. Soil was sandy and alkaline, with a relatively low content of total and/or exchangeable forms of many studied elements, both nutrients and xenobiotics (C, K, Mg, P, Pb, Tl and Zn). This substratum was characteristic for the most xerothermic habitat of the studied area, covered mainly by Agrostis stolonifera, Calamagrostis epigejos, Melilotus officinalis and Solidago canadensis (Tab. 1, site 9).

\section{Culm substratum}

A substratum consisting of culm left after coal production. Because it is not very permeable, numerous shallow water pools have come into being. Sandy clay soil was slightly acidic and contained the highest percent of carbon among all the tested soils. Moreover, concentrations of exchangeable forms of $\mathrm{K}, \mathrm{Mg}, \mathrm{Mn}$ and $\mathrm{Na}$ were elevated. However, the soil was poor with respect to $\mathrm{Ca}$ and available $\mathrm{P}$ content. Juncus tenuis, Phragmites australis, Typha latifolia and T. laxmannii were dominant plant species growing at this site (Tab. 1, site 8).

\section{Humus substratum}

Initial soil developed on the oldest and poorly disturbed (even during mine operation) part of carboniferous waste rock spoil, under 20-30 year old trees of Betula pendula, Pinus sylvestris, Populus tremula and Salix caprea. Sand or loamy sand soils were alkaline, although total $\mathrm{Ca}$ and $\mathrm{Mg}$ contents were diverse. Additionally, total Mn content was the highest among all tested soils. Calluna vulgaris, Deschampsia flexuosa and Vaccinium myrtillus dominated the undergrowth (Tab. 1, sites 7 and 10).

\section{Illegal dumps of construction debris with rubbish}

Unstable substratum of concrete-brick debris originating from building demolition mixed with household rubbish, resulting from the illegal activities of the local people. Alkaline sandy loam or loamy sand contained relatively low amounts of total $\mathrm{C}$ and $\mathrm{Fe}$. Total $\mathrm{Ca}$ and $\mathrm{P}$ contents were relatively high, while $\mathrm{N}$ content was diverse. It was covered mainly with annual plant species such as Chenopodium album, species from Polygonum genus $(P$. aviculare, P. persicaria) and Setaria viridis (Tab. 1, sites 1 and 3).

\section{Coal ashes}

Deposited separately, unstable mounds of ashes after coal burning in the nearby Siersza power plant. Sandy loam soil developed on the site was alkaline and had the highest total Fe, $\mathrm{Mg}$, Na content among studied soils, and relatively high $\mathrm{S}$, total $\mathrm{P}$ and water-soluble $\mathrm{Pb}$ content. On the other hand, $\mathrm{N}$ and exchangeable Ca levels were relatively low. The site was cov- 
ered mainly with grasses such as Agrostis stolonifera, Calamagrostis epigejos and Poa compressa (Tab. 1, site 6).

\section{Floristic properties}

The number of plant species per $4 \mathrm{~m}^{2}$ varied from 6 to 29 , and plant cover from 50 to $95 \%$. The highest number of species per area unit was observed on humus substratum, on illegal dumps of construction debris with rubbish and on a mixed substratum. The number of plant species decreased with increasing total carbon content in the substratum $(\mathrm{r}=-0.85, \mathrm{p}<$ 0.01; Fig. 2) and increased with sand content ( $\mathrm{r}=0.66, \mathrm{p}<0.05$; Fig. 3). High carbon and clay contents (relatively low sand content) are related with coal and culm substrata (Tab. 1). Culm material (site 8) and coal substratum (sites 2 and 4) were found to be unfavorable for vegetation. The only exception was coal site 5 , characterized by a relatively high nutrient content $(\mathrm{N}, \mathrm{S}, \mathrm{Mg}, \mathrm{P})$ and high number of species.

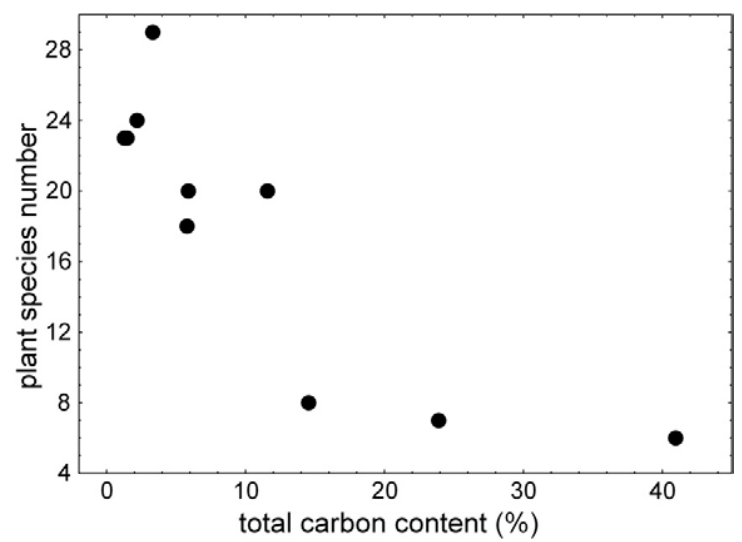

Fig. 2. Correlation between total carbon content in substratum and a number of plant species (Spearman correlation: $\mathrm{r}=-0.85, \mathrm{p}<0.01$ ).

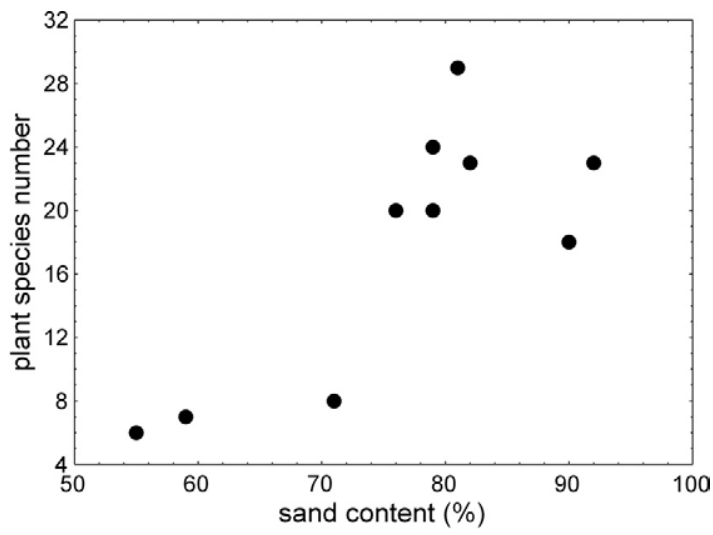

Fig. 3. Correlation between sand content in substratum and a number of plant species (Spearman correlation: $r=0.66, p<0.05)$. 
In the approximately 20 ha of the studied area of the former Siersza hard coal mine, 197 species of plants belonging to 53 families were reported (Tab. 2). The highest number of species belonged to Asteraceae (36), followed by Fabaceae (19), Poaceae (17), Rosaceae (16), Polygonaceae (8), Scrophulariaceae (7), Salicaceae (7), Brassicaceae (6), Lamiaceae (6), Apiaceae (5), Caryophyllaceae (5). Some families were represented by 2 or 3 species: Aceraceae (3), Betulaceae (3), Dipsacaceae (3), Euphorbiaceae (3), Balsaminaceae (2), Boraginaceae (2), Caprifoliaceae (2), Ericaceae (2), Fagaceae (2), Juncaceae (2), Onagraceae (2), Orchidaceae (2), Papaveraceae (2), Pinaceae (2), Plantaginaceae (2), Ranunculaceae (2), Typhaceae (2), Violaceae (2). A lot of families were represented by 1 species: Amaranthaceae, Anacardiaceae, Chenopodiaceae, Clusiaceae, Convolvulaceae, Cornaceae, Corylaceae, Crassulaceae, Cyperaceae, Equisetaceae, Oleaceae, Geraniaceae, Hippocastanaceae, Hypolepidaceae, Liliaceae, Linaceae, Malvaceae, Oxalidaceae, Resedaceae, Rhamnaceae, Rubiaceae, Solanaceae, Urticaceae and Vitaceae. As far as life forms are concerned, it was observed that hemicryptophytes predominated (49\%). Second group were therophytes (18\%). Quite a few were also mega-phanerophytes (10\%), geophytes (9\%) and nano-phanerophytes $(7 \%)$. The remaining species $(3 \%)$ belonged to non-ligneous chamaephytes, ligneous chamaephytes and hydrophytes (2\% each), 1 species was a liana (Fig. 4). Despite the prevalence of native species $(69 \%)$, one third of the flora was represented by alien plants (anthropophytes) (31\%). Among anthropophytes the most numerous were archaeophytes (plants that were introduced up to the end of the 15th century) (13\%). The next groups of alien species were: kenophytes (newcomers, after 15th century) (8\%), epecophytes (established only in ruderal and/or segetal communities) (4\%), hemiagriophytes (established in semi-natural communities) (1\%), agriophytes (established in natural

Tab. 2. List of plant species occurring in the study area. Substratum: $\mathbf{C c}-$ carboniferous shale with coal, $\mathbf{C o}$ - coal ashes, $\mathbf{C m}$ - culm substratum, $\mathbf{D}$ - illegal dumps of construction debris with rubbish, $\mathbf{H u}$ - humus substratum, $\mathbf{M x}$ - mixed substratum (see Tab. 1), a order of substratum short-cuts means a descending frequency of species records on a given substratum; Form life forms from Raunkiaer: $\mathbf{G}$ - geophyte, $\mathbf{H}$ - hemicryptophyte, $\mathbf{M}$ - megafanerophyte, $\mathbf{T}$ therophyte, $\mathbf{C}$ - non-ligneous chamaephyte, $\mathbf{C h}$ - ligneous chamaephyte, $\mathbf{H y}$ - hydrophyte, $\mathbf{N}$ - nano-phanerophyte, $\mathbf{L}$ - liana. Geographic-historical classification in Polish flora: $\mathbf{R}$ native species, $\mathbf{K}$ - kenophyte, $\mathbf{A}$ - archaeophyte, Hemi - hemiagriophyte, $\mathbf{A g r}$ - agriophyte, Erg - ergaziophyte, Ep - epecophyte, Ef - ephemerophyte, dispersion - types of seed dispersion: An - anemochory, Zooch - zoochory, Hy - hydrochory, Antr - anthropochory, Au - autochory, B - barachory; juv. - juvenile specimen.

\begin{tabular}{lccccc}
\hline Species & $\begin{array}{c}\text { No of } \\
\text { records }\end{array}$ & Substratum & Form & Status & Dispersion \\
\hline Acer campestre (juv.) & 31 & $\mathrm{Mx}, \mathrm{D}$ & $\mathrm{M}$ & $\mathrm{R}$ & $\mathrm{An}$ \\
Acer negundo (juv.) & 35 & $\mathrm{Mx}, \mathrm{D}$ & $\mathrm{M}$ & $\mathrm{K}$ & $\mathrm{An}$ \\
Acer platanoides (juv.) & 34 & $\mathrm{Mx}, \mathrm{Hu}$ & $\mathrm{M}$ & $\mathrm{R}$ & $\mathrm{An}$ \\
Achillea millefolium & 144 & $\mathrm{Mx}, \mathrm{D}$ & $\mathrm{H}$ & $\mathrm{R}$ & Zooch \\
Acinos arvensis & 45 & $\mathrm{Mx}, \mathrm{D}$ & $\mathrm{T} / \mathrm{H}$ & $\mathrm{R}$ & An \\
Aegopodium podagraria & 31 & $\mathrm{D}$ & $\mathrm{G} / \mathrm{H}$ & $\mathrm{R}$ & Zooch \\
Aesculus hippocastanum (juv.) & 1 & $\mathrm{Mx}$ & $\mathrm{M}$ & $\mathrm{K}$ & Zooch/B \\
Agrostis gigantea & 15 & $\mathrm{Mx}, \mathrm{D}, \mathrm{Cc}$ & $\mathrm{H}$ & $\mathrm{R}$ & $\mathrm{An}$ \\
\hline
\end{tabular}


Tab. 2. - continued

\begin{tabular}{|c|c|c|c|c|c|}
\hline Species & $\begin{array}{l}\text { No of } \\
\text { records }\end{array}$ & Substratum & Form & Status & Dispersion \\
\hline Agrostis stolonifera & 159 & $\mathrm{Mx}, \mathrm{D}, \mathrm{Hu}, \mathrm{Cm}$ & $\mathrm{H}$ & $\mathrm{R}$ & An \\
\hline Alnus glutinosa (juv.) & 1 & $\mathrm{Hu}$ & M & $\mathrm{R}$ & An \\
\hline Alnus incana (juv.) & 8 & $\mathrm{Cc}$ & M & $\mathrm{R}$ & An \\
\hline Amaranthus retroflexus & 2 & $\mathrm{D}$ & $\mathrm{T}$ & Ep & An \\
\hline Anthemis arvensis & 67 & $\mathrm{D}$ & $\mathrm{T}$ & A & Zooch \\
\hline Anthemis tinctoria & 1 & $\mathrm{Mx}$ & $\mathrm{H}$ & $\mathrm{R}$ & Zooch \\
\hline Antirrhinum majus & 1 & $\mathrm{D}, \mathrm{Cc}$ & $\mathrm{T}$ & Ef & An/Antr \\
\hline Arctium lappa & 1 & $\mathrm{Mx}$ & $\mathrm{H}$ & $\mathrm{R}$ & Zooch \\
\hline Artemisia absinthium & 3 & $\mathrm{Mx}$ & $\mathrm{Ch}$ & A & An \\
\hline Artemisia vulgaris & 99 & $\mathrm{Mx}, \mathrm{D}$ & $\mathrm{C}$ & $\mathrm{R}$ & An \\
\hline Astragalus glycyphyllos & 12 & $\mathrm{Mx}, \mathrm{Hu}$ & $\mathrm{H}$ & $\mathrm{R}$ & Zooch \\
\hline Bellis perennis & 2 & $\mathrm{D}, \mathrm{Cc}$ & $\mathrm{H}$ & $\mathrm{R}$ & Zooch \\
\hline Berteroa incana & 1 & $\mathrm{Mx}, \mathrm{D}$ & $\mathrm{T} / \mathrm{H}$ & A & An \\
\hline Betula pendula & 130 & $\mathrm{Mx}, \mathrm{Cc}, \mathrm{Hu}$ & M & $\mathrm{R}$ & An \\
\hline Bidens frondosa & 63 & $\mathrm{Cm}, \mathrm{D}$ & $\mathrm{T}$ & Agr & $\begin{array}{c}\text { An/Antr/ } \\
\text { Hy/Zooch }\end{array}$ \\
\hline Brachypodium pinnatum & 25 & $\mathrm{Cc}, \mathrm{Mx}$ & $\mathrm{H} / \mathrm{C}$ & $\mathrm{R}$ & An \\
\hline Bromus hordeaceus & 11 & Mx & $\mathrm{T}$ & $\mathrm{R}$ & An \\
\hline Bromus tectorum & 4 & $\mathrm{Mx}, \mathrm{D}, \mathrm{Cc}$ & $\mathrm{T}$ & A & An \\
\hline Calamagrostis epigejos & 179 & $\mathrm{Mx}, \mathrm{D}, \mathrm{Hu}, \mathrm{Cc}, \mathrm{Cm}$ & $\mathrm{G} / \mathrm{H}$ & $\mathrm{R}$ & An \\
\hline Calendula officinalis & 4 & $\mathrm{D}$ & $\mathrm{T}$ & Erg & Zooch \\
\hline Calluna vulgaris & 9 & $\mathrm{Hu}, \mathrm{Mx}, \mathrm{Cc}$ & $\mathrm{Ch}$ & $\mathrm{R}$ & An \\
\hline Capsella bursa-pastoris & 18 & Mx, D & $\mathrm{T} / \mathrm{H}$ & $\mathrm{A}$ & An \\
\hline Cardaminopsis arenosa & 44 & $\mathrm{Mx}, \mathrm{D}$ & $\mathrm{H} / \mathrm{T}$ & $\mathrm{R}$ & An \\
\hline Carex hirta & 14 & $\mathrm{D}, \mathrm{Mx}$ & $\mathrm{G}$ & $\mathrm{R}$ & An \\
\hline Carlina vulgaris & 1 & $\mathrm{Hu}$ & $\mathrm{H}$ & $\mathrm{R}$ & An/Zooch \\
\hline Carpinus betulus & 2 & $\mathrm{Hu}$ & M & $\mathrm{R}$ & An \\
\hline Cenaturea jacea & 63 & $\mathrm{Mx}, \mathrm{D}, \mathrm{Hu}$ & $\mathrm{H}$ & $\mathrm{R}$ & An \\
\hline Cenaturea stoebe & 67 & $\mathrm{Mx}, \mathrm{D}$ & $\mathrm{H}$ & $\mathrm{R}$ & An \\
\hline Chamaecytisus ratisbonensis & 3 & Mx & $\mathrm{Ch} / \mathrm{N}$ & $\mathrm{R}$ & Zooch \\
\hline Chamaenerion angustifolium & 6 & $\mathrm{D}, \mathrm{Hu}, \mathrm{Mx}$ & $\mathrm{H}$ & $\mathrm{R}$ & An \\
\hline Chamomilla recutita & 10 & $\mathrm{Mx}, \mathrm{D}, \mathrm{Hu}, \mathrm{Cc}$ & $\mathrm{T}$ & A & An \\
\hline Chamomilla suaveolens & 13 & $\mathrm{Mx}, \mathrm{Cc}$ & $\mathrm{T}$ & $\mathrm{K}$ & An \\
\hline Chelidonium majus & 27 & $\mathrm{D}$ & $\mathrm{H}$ & $\mathrm{R}$ & Zooch \\
\hline Chenopodium album & 47 & $\mathrm{D}, \mathrm{Cc}$ & $\mathrm{T}$ & $\mathrm{R}$ & An \\
\hline Cichorium intybus & 73 & $\mathrm{Mx}, \mathrm{D}, \mathrm{Hu}, \mathrm{Cc}, \mathrm{Co}$ & $\mathrm{H}$ & A & An \\
\hline Cirsium vulgare & 97 & $\mathrm{Mx}, \mathrm{D}, \mathrm{Hu}$ & $\mathrm{H}$ & $\mathrm{R}$ & An/Zooch \\
\hline Clinopodium vulgare & 52 & $\mathrm{Mx}$ & $\mathrm{H}$ & $\mathrm{R}$ & An \\
\hline
\end{tabular}


Woch M. W., Radwańska M., Stefanowicz A. M.

Tab. 2. - continued

\begin{tabular}{|c|c|c|c|c|c|}
\hline Species & $\begin{array}{l}\text { No of } \\
\text { records }\end{array}$ & Substratum & Form & Status & Dispersion \\
\hline Convallaria majalis & 6 & Mx, D & G & $\mathrm{R}$ & Zooch \\
\hline Convolvulus arvensis & 2 & $\mathrm{Mx}, \mathrm{Cc}$ & $\mathrm{H}$ & $\mathrm{R}$ & An/Antr \\
\hline Conyza canadensis & 111 & $\mathrm{Mx}, \mathrm{D}, \mathrm{Co}, \mathrm{Co}$ & $\mathrm{T} / \mathrm{H}$ & Ep & An \\
\hline Cornus alba & 4 & $\mathrm{Mx}$ & $\mathrm{N}$ & $\mathrm{R}$ & Zooch \\
\hline Coronilla varia & 73 & $\mathrm{Mx}, \mathrm{Cc}$ & $\mathrm{H}$ & $\mathrm{R}$ & $\mathrm{Au}$ \\
\hline Corynephorus canescens & 24 & $\mathrm{Mx}, \mathrm{D}$ & $\mathrm{H}$ & $\mathrm{R}$ & An \\
\hline Crataegus monogyna & 1 & $\mathrm{Hu}$ & $\mathrm{N} / \mathrm{M}$ & $\mathrm{R}$ & Zooch \\
\hline Dactylis glomerata & 12 & $\mathrm{Mx}$ & $\mathrm{H}$ & $\mathrm{R}$ & An \\
\hline Daucus carota & 127 & Mx & $\mathrm{H}$ & $\mathrm{R}$ & Zooch \\
\hline Deschampsia flexuosa & 2 & $\mathrm{Hu}$ & $\mathrm{H}$ & $\mathrm{R}$ & An \\
\hline Dianthus deltoides & 2 & Mx & $\mathrm{H} / \mathrm{C}$ & $\mathrm{R}$ & An \\
\hline Dipsacus fullonum & 1 & $\mathrm{Mx}$ & $\mathrm{H}$ & A & An \\
\hline $\mathrm{t}$ Echium vulgare & 71 & $\mathrm{Mx}, \mathrm{D}, \mathrm{Cc}$ & $\mathrm{H}$ & A & An/Zooch \\
\hline Elymus repens & 42 & $\mathrm{Mx}, \mathrm{D}, \mathrm{Cc}$ & G & $\mathrm{R}$ & An \\
\hline Epipactis atrorubens & 1 & $\mathrm{Hu}$ & G & $\mathrm{R}$ & An \\
\hline Epipactis helleborine & 3 & $\mathrm{Hu}$ & G & $\mathrm{R}$ & An \\
\hline Equisetum arvense & 32 & $\mathrm{Mx}, \mathrm{Hu}, \mathrm{D}$ & G & $\mathrm{R}$ & An \\
\hline Erigeron annuus & 105 & $\mathrm{Mx}, \mathrm{D}, \mathrm{Hu}, \mathrm{Cc}, \mathrm{Cm}$ & $\mathrm{H}$ & $\mathrm{K}$ & An \\
\hline Erysimum cheiranthoides & 13 & $\mathrm{D}, \mathrm{Cc}$ & $\mathrm{T}$ & A & An \\
\hline Eupatorium cannabinum & 91 & $\mathrm{D}, \mathrm{Co}$ & $\mathrm{H}$ & $\mathrm{R}$ & An \\
\hline Euphorbia cyparissias & 33 & $\mathrm{Mx}, \mathrm{Hu}$ & $\mathrm{H}$ & $\mathrm{R}$ & Zooch \\
\hline Euphorbia esula & 1 & $\mathrm{D}$ & $\mathrm{H}$ & $\mathrm{R}$ & Zooch \\
\hline Euphorbia helioscopia & 2 & $\mathrm{D}$ & $\mathrm{T}$ & A & Zooch \\
\hline Euphrasia rostkoviana & 5 & $\mathrm{Hu}, \mathrm{Mx}$ & $\mathrm{T}$ & $\mathrm{R}$ & An \\
\hline Fallopia convolvulus & 16 & $\mathrm{D}$ & $\mathrm{T}$ & A & An \\
\hline Fragaria vesca & 60 & $\mathrm{Mx}, \mathrm{Cc}$ & $\mathrm{H}$ & $\mathrm{R}$ & Zooch \\
\hline Fragaria $\mathrm{x}$ ananassa & 1 & $\mathrm{D}$ & $\mathrm{H}$ & Ef & Zooch \\
\hline Frangula alnus & 1 & $\mathrm{Hu}$ & $\mathrm{N}$ & $\mathrm{R}$ & Zooch \\
\hline Galeopsis tetrahit & 1 & $\mathrm{D}$ & $\mathrm{T}$ & $\mathrm{R}$ & An \\
\hline Galinsoga parviflora & 6 & $\mathrm{D}$ & $\mathrm{T}$ & K & An \\
\hline Galium mollugo & 18 & $\mathrm{Mx}$ & $\mathrm{H}$ & $\mathrm{R}$ & An \\
\hline Genista germanica & 4 & Mx & $\mathrm{N}$ & $\mathrm{R}$ & Zooch \\
\hline Geranium pratense & 1 & $\mathrm{Hu}$ & $\mathrm{H}$ & $\mathrm{R}$ & $\mathrm{Au}$ \\
\hline Geum urbanum & 4 & $\mathrm{Mx}, \mathrm{D}$ & $\mathrm{H}$ & $\mathrm{R}$ & Zooch \\
\hline Helianthus annuus & 1 & $\mathrm{Mx}$ & $\mathrm{H}$ & Ef & Zooch \\
\hline Heracleum sphondylium & 32 & $\mathrm{Hu}, \mathrm{D}$ & $\mathrm{H}$ & $\mathrm{R}$ & Zooch \\
\hline Hieracium vulgatum & 2 & Mx & $\mathrm{H}$ & $\mathrm{R}$ & An \\
\hline Hieracium pilosella & 12 & $\mathrm{Mx}, \mathrm{Hu}$ & $\mathrm{H}$ & $\mathrm{R}$ & Zooch \\
\hline
\end{tabular}


Tab. 2. - continued

\begin{tabular}{|c|c|c|c|c|c|}
\hline Species & $\begin{array}{l}\text { No of } \\
\text { records }\end{array}$ & Substratum & Form & Status & Dispersion \\
\hline Holcus mollis & 1 & Mx & $\mathrm{H} / \mathrm{G}$ & $\mathrm{R}$ & An \\
\hline Hypericum perforatum & 96 & $\mathrm{Mx}, \mathrm{Hu}, \mathrm{D}$ & $\mathrm{H}$ & $\mathrm{R}$ & An/Zooch \\
\hline Impatiens glandulifera & 2 & $\mathrm{D}$ & $\mathrm{T}$ & $\mathrm{K}$ & $\mathrm{Au}$ \\
\hline Impatiens parviflora & 7 & $\mathrm{Mx}, \mathrm{Cc}, \mathrm{D}$ & $\mathrm{T}$ & $\mathrm{K}$ & $\mathrm{Au}$ \\
\hline Juncus conglomeratus & 7 & $\mathrm{Cm}, \mathrm{Mx}$ & $\mathrm{H}$ & $\mathrm{R}$ & An/Zooch \\
\hline Juncus tenuis & 4 & $\mathrm{Cm}, \mathrm{Mx}, \mathrm{D}$ & $\mathrm{H}$ & K & An/Zooch \\
\hline Knautia arvensis & 7 & $\mathrm{Mx}$ & $\mathrm{H}$ & $\mathrm{R}$ & An \\
\hline Lactuca serriola & 6 & $\mathrm{Mx}, \mathrm{Hu}$ & $\mathrm{H}$ & A & An \\
\hline Lamium album & 2 & $\mathrm{Cc}, \mathrm{D}$ & $\mathrm{H}$ & A & Zooch \\
\hline Larix decidua & 1 & $\mathrm{D}$ & M & $\mathrm{R}$ & An \\
\hline Lathyrus pratensis & 1 & $\mathrm{Hu}$ & $\mathrm{H}$ & $\mathrm{R}$ & $\mathrm{Au}$ \\
\hline Leontodon hispidus & 33 & $\mathrm{Mx}, \mathrm{Cc}$ & $\mathrm{H}$ & $\mathrm{R}$ & An \\
\hline Ligustrum vulgare & 2 & $\mathrm{Mx}$ & $\mathrm{N}$ & $\mathrm{K}$ & Zooch \\
\hline Linum catharticum & 2 & Mx & $\mathrm{T}$ & $\mathrm{H}$ & $\mathrm{R}$ \\
\hline Lolium perenne & 4 & $\mathrm{Mx}, \mathrm{D}, \mathrm{Cc}$ & $\mathrm{H}$ & $\mathrm{R}$ & An \\
\hline Lotus corniculatus & 143 & $\mathrm{Mx}, \mathrm{Hu}, \mathrm{D}, \mathrm{Cc}$ & $\mathrm{H}$ & $\mathrm{R}$ & $\mathrm{Au}$ \\
\hline Lupinus polyphyllus & 1 & $\mathrm{Mx}$ & $\mathrm{H}$ & Ep & $\mathrm{Au}$ \\
\hline Malva alcea & 1 & Mx & $\mathrm{H}$ & A & $\mathrm{Au}$ \\
\hline Medicago lupulina & 141 & $\mathrm{Mx}, \mathrm{D}$ & $\mathrm{T}$ & $\mathrm{H}$ & $\mathrm{R}$ \\
\hline Medicago sativa & 4 & $\mathrm{D}, \mathrm{Mx}$ & $\mathrm{H}$ & $\mathrm{K}$ & $\mathrm{Au}$ \\
\hline Melampyrum pratense & 2 & $\mathrm{Mx}$ & $\mathrm{T}$ & $\mathrm{R}$ & An \\
\hline Melandrium album & 66 & $\mathrm{Mx}, \mathrm{D}, \mathrm{Co}$ & $\mathrm{T}$ & $\mathrm{R}$ & An \\
\hline Melilotus alba & 114 & $\mathrm{Mx}, \mathrm{D}, \mathrm{Cc}$ & $\mathrm{H} / \mathrm{T}$ & A & An/Zooch \\
\hline Melilotus officinalis & 103 & $\mathrm{Mx}, \mathrm{D}, \mathrm{Cc}$ & $\mathrm{H}$ & A & An/Zooch \\
\hline Molinia caerulea & 67 & $\mathrm{Mx}$ & $\mathrm{H}$ & $\mathrm{R}$ & An \\
\hline Myosoton aquaticum & 1 & $\mathrm{Hu}$ & G & $\mathrm{R}$ & An \\
\hline mOdontites serotina & 4 & Mx & $\mathrm{T}$ & $\mathrm{R}$ & An \\
\hline Oenothera biennis & 112 & $\mathrm{Mx}, \mathrm{D}$ & $\mathrm{H}$ & $\mathrm{K}$ & An \\
\hline Ononis spinosa & 1 & Mx & $\mathrm{H}$ & $\mathrm{R}$ & $\mathrm{Au}$ \\
\hline Origanum vulgare & 27 & Mx & $\mathrm{H}$ & $\mathrm{C}$ & Zooch \\
\hline Oxalis europaea & 2 & $\mathrm{D}$ & G & Ep & $\mathrm{Au}$ \\
\hline Papaver rhoeas & 26 & $\mathrm{D}, \mathrm{Mx}$ & $\mathrm{T}$ & A & An \\
\hline Parthenocissus inserta & 1 & $\mathrm{Mx}$ & $\mathrm{L}$ & Ef & Antr \\
\hline Pastinaca sativa & 96 & $\mathrm{Mx}, \mathrm{D}, \mathrm{Cc}$ & $\mathrm{H}$ & $\mathrm{R}$ & Zooch \\
\hline Phragmites australis & 129 & $\mathrm{Cm}, \mathrm{D}, \mathrm{Cc}$ & G/Hy & $\mathrm{R}$ & An \\
\hline Picris hieracioides & 91 & $\mathrm{Mx}, \mathrm{Cc}$ & $\mathrm{H}$ & $\mathrm{R}$ & An \\
\hline Pinus sylvestris & 145 & $\mathrm{Mx}, \mathrm{Hu}, \mathrm{D}, \mathrm{Cc}$ & M & $\mathrm{R}$ & An \\
\hline Plantago lanceolata & 82 & $\mathrm{D}, \mathrm{Mx}$ & $\mathrm{H}$ & $\mathrm{R}$ & Zooch \\
\hline
\end{tabular}


Woch M. W., Radwańska M., Stefanowicz A. M.

Tab. 2. - continued

\begin{tabular}{|c|c|c|c|c|c|}
\hline Species & $\begin{array}{l}\text { No of } \\
\text { records }\end{array}$ & Substratum & Form & Status & Dispersion \\
\hline Plantago major & 98 & $\mathrm{D}, \mathrm{Mx}$ & $\mathrm{H}$ & $\mathrm{R}$ & Zooch \\
\hline Poa compressa & 105 & $\mathrm{Mx}, \mathrm{Cc}, \mathrm{D}, \mathrm{Hu}$ & $\mathrm{H}$ & $\mathrm{R}$ & An \\
\hline Poa nemoralis & 1 & $\mathrm{Hu}$ & $\mathrm{H}$ & $\mathrm{R}$ & An \\
\hline Polygonum aviculare & 31 & $\mathrm{D}$ & $\mathrm{T}$ & $\mathrm{R}$ & An/Antr \\
\hline Polygonum lapathifolium & 12 & $\mathrm{Cc}, \mathrm{D}$ & $\mathrm{T}$ & $\mathrm{R}$ & An/Antr \\
\hline Polygonum persicaria & 68 & $\mathrm{Cc}, \mathrm{D}$ & $\mathrm{T}$ & $\mathrm{R}$ & An/Antr \\
\hline Populus nigra 'italica' & 4 & $\mathrm{Cc}, \mathrm{D}$ & M & $\mathrm{R}$ & An \\
\hline Populus tremula & 129 & $\mathrm{Mx}, \mathrm{Cc}$ & M & $\mathrm{R}$ & An \\
\hline Populus wilsonii & 1 & Mx & M & Erg & An \\
\hline Populus x canadensis & 1 & Mx & M & Erg & An \\
\hline Potentilla anserina & 17 & $\mathrm{Mx}, \mathrm{D}, \mathrm{Co}$ & $\mathrm{H}$ & $\mathrm{R}$ & An \\
\hline Potentilla argentea & 2 & $\mathrm{Mx}$ & $\mathrm{H}$ & $\mathrm{R}$ & An \\
\hline Potentilla reptans & 2 & $\mathrm{Mx}$ & $\mathrm{H}$ & $\mathrm{R}$ & An \\
\hline Potentilla supina & 28 & $\mathrm{Mx}$ & $\mathrm{T} / \mathrm{H}$ & $\mathrm{R}$ & An \\
\hline Prunella vulgaris & 63 & Mx & $\mathrm{H}$ & $\mathrm{R}$ & Hy/Zooch \\
\hline Prunus avium & 3 & $\mathrm{Hu}$ & $\mathrm{M} / \mathrm{N}$ & $\mathrm{R}$ & Zooch \\
\hline Prunus serotina & 2 & $\mathrm{Hu}$ & $\mathrm{M}$ & Agr & Zooch \\
\hline Prunus spinosa & 1 & $\mathrm{Hu}$ & $\mathrm{N}$ & $\mathrm{R}$ & Zooch \\
\hline Pteridium aquilinum & 14 & $\mathrm{Hu}$ & G & $\mathrm{R}$ & An \\
\hline Quercus petraea & 6 & $\mathrm{Hu}$ & M & $\mathrm{R}$ & Zooch \\
\hline Quercus rubra & 29 & $\mathrm{Hu}$ & M & Hemi & Zooch \\
\hline Ranunculus acris & 3 & $\mathrm{D}, \mathrm{Hu}$ & $\mathrm{H}$ & $\mathrm{R}$ & $\mathrm{Au}$ \\
\hline Ranunculus bulbosus & 1 & $\mathrm{Mx}$ & G & $\mathrm{H}$ & $\mathrm{Au}$ \\
\hline Reseda lutea & 16 & $\mathrm{D}, \mathrm{Mx}$ & $\mathrm{H}$ & $\mathrm{K}$ & An \\
\hline Rhus typhina & 1 & $\mathrm{Cc}$ & $\mathrm{M} / \mathrm{N}$ & Erg & Zooch \\
\hline Robinia pseudacacia & 136 & $\mathrm{Mx}, \mathrm{Cc}$ & $\mathrm{M}$ & Hemi & An/Zooch \\
\hline Rosa canina & 3 & $\mathrm{Mx}$ & $\mathrm{N}$ & $\mathrm{R}$ & Zooch \\
\hline Rubus ceasius & 64 & $\mathrm{Mx}, \mathrm{D}, \mathrm{Hu}$ & $\mathrm{N} / \mathrm{Ch}$ & $\mathrm{R}$ & Zooch \\
\hline Rumex acetosa & 14 & $\mathrm{Mx}, \mathrm{D}, \mathrm{Hu}$ & $\mathrm{H}$ & $\mathrm{R}$ & An \\
\hline Rumex acetosella & 8 & $\mathrm{Mx}, \mathrm{D}, \mathrm{Co}$ & $\mathrm{G} / \mathrm{H}$ & $\mathrm{R}$ & An \\
\hline Rumex obtusifolius & 3 & Mx, Co & $\mathrm{H}$ & $\mathrm{K}$ & An \\
\hline Rumex thyrsiflorus & 4 & $\mathrm{Mx}, \mathrm{Co}, \mathrm{Cc}$ & $\mathrm{H}$ & $\mathrm{R}$ & An \\
\hline Salix alba & 66 & $\mathrm{Mx}, \mathrm{Cc}, \mathrm{D}$ & M & $\mathrm{R}$ & An \\
\hline Salix caprea & 75 & $\mathrm{Mx}, \mathrm{Cc}, \mathrm{D}$ & $\mathrm{N} / \mathrm{M}$ & $\mathrm{R}$ & An \\
\hline rin0Salix cinerea & 1 & $\mathrm{Hu}$ & $\mathrm{N}$ & $\mathrm{R}$ & An \\
\hline Sambucus nigra & 2 & $\mathrm{Hu}$ & $\mathrm{N} / \mathrm{M}$ & $\mathrm{R}$ & Zooch \\
\hline Sanguisorba officinalis & 1 & $\mathrm{Cc}$ & $\mathrm{H}$ & $\mathrm{R}$ & Zooch \\
\hline Scabiosa ochroleuca & 8 & $\mathrm{Mx}$ & $\mathrm{H}$ & $\mathrm{R}$ & An \\
\hline
\end{tabular}


Tab. 2. - continued

\begin{tabular}{|c|c|c|c|c|c|}
\hline Species & $\begin{array}{l}\text { No of } \\
\text { records }\end{array}$ & Substratum & Form & Status & Dispersion \\
\hline Sedum acre & 12 & Mx & $\mathrm{C}$ & $\mathrm{R}$ & Hy/Zooch \\
\hline Senecio jacobaea & 14 & $\mathrm{Hu}, \mathrm{Cc}, \mathrm{D}$ & $\mathrm{H}$ & $\mathrm{R}$ & An \\
\hline Senecio vulgaris & 1 & D & $\mathrm{T} / \mathrm{H}$ & A & An \\
\hline Setaria viridis & 29 & $\mathrm{D}, \mathrm{Mx}, \mathrm{Cc}$ & $\mathrm{T}$ & A & An \\
\hline Silene vulgaris & 74 & $\mathrm{Mx}, \mathrm{Cc}, \mathrm{D}, \mathrm{Co}$ & $\mathrm{H} / \mathrm{C}$ & $\mathrm{R}$ & An \\
\hline Sinapis arvensis & 5 & $\mathrm{D}, \mathrm{Mx}$ & $\mathrm{T}$ & $\mathrm{A}$ & An \\
\hline Sisymbrium loeselii & 3 & D & $\mathrm{T} / \mathrm{H}$ & Ep & An \\
\hline Solanum tuberosum & 1 & $\mathrm{D}$ & $\mathrm{G}$ & Ef & Zooch \\
\hline Solidago canadensis & 183 & $\mathrm{Mx}, \mathrm{Hu}, \mathrm{D}$ & $\mathrm{H} / \mathrm{G}$ & Agr & An \\
\hline Solidago gigantea & 1 & $\mathrm{Mx}$ & $\mathrm{H} / \mathrm{G}$ & Agr & $\mathrm{Am}$ \\
\hline Solidago virgaurea & 3 & $\mathrm{Cc}$ & $\mathrm{H}$ & $\mathrm{R}$ & An \\
\hline Sonchus asper & 28 & $\mathrm{Mx}, \mathrm{D}$ & $\mathrm{T}$ & $\mathrm{A}$ & An \\
\hline Sorbus aucuparia & 3 & $\mathrm{Hu}$ & M & $\mathrm{R}$ & Zooch \\
\hline Spiraea media & 1 & $\mathrm{Hu}$ & $\mathrm{N}$ & $\mathrm{R}$ & An \\
\hline Stellaria graminea & 12 & $\mathrm{Mx}, \mathrm{Hu}$ & $\mathrm{H}$ & $\mathrm{R}$ & An \\
\hline Symphoricarpos albus & 3 & $\mathrm{Hu}$ & $\mathrm{N}$ & Ef & Zooch \\
\hline Symphytum officinale & 2 & $\mathrm{Cc}, \mathrm{D}$ & $\mathrm{H}$ & $\mathrm{R}$ & An/Antr \\
\hline Tanacetum vulgare & 148 & $\mathrm{Mx}, \mathrm{Cc}, \mathrm{D}, \mathrm{Hu}$ & $\mathrm{H}$ & $\mathrm{R}$ & An \\
\hline Taraxacum officinale & 138 & $\mathrm{Mx}, \mathrm{Cc}, \mathrm{D}$ & $\mathrm{H}$ & Ep & An \\
\hline Torilis japonica & 31 & $\mathrm{Hu}$ & $\mathrm{T} / \mathrm{H}$ & $\mathrm{R}$ & Zooch \\
\hline Tragopogon pratensis & 1 & $\mathrm{Cc}$ & $\mathrm{H}$ & Ep & An \\
\hline Trifolium arvense & 31 & $\mathrm{Mx}, \mathrm{Cc}$ & $\mathrm{T}$ & $\mathrm{R}$ & An/Zooch \\
\hline Trifolium medium & 4 & Mx & $\mathrm{H}$ & $\mathrm{R}$ & An/Zooch \\
\hline Trifolium pratense & 137 & $\mathrm{Mx}, \mathrm{D}$ & $\mathrm{H}$ & $\mathrm{R}$ & An \\
\hline Trifolium repens & 51 & Mx & $\mathrm{H}$ & $\mathrm{R}$ & Zooch \\
\hline Tussilago farfara & 144 & $\mathrm{Mx}, \mathrm{Cc}, \mathrm{Co}, \mathrm{Cm}$ & $\mathrm{G} / \mathrm{H}$ & $\mathrm{R}$ & An \\
\hline Typha latifolia & 16 & $\mathrm{Cm}, \mathrm{Cc}$ & $\mathrm{Hy} / \mathrm{H}$ & $\mathrm{R}$ & An \\
\hline Typha laxmannii & 3 & $\mathrm{Cm}$ & $\mathrm{Hy} / \mathrm{H}$ & $\mathrm{K}$ & An \\
\hline Urtica dioica & 44 & $\mathrm{D}, \mathrm{Mx}, \mathrm{Hu}, \mathrm{Cc}$ & $\mathrm{H}$ & $\mathrm{R}$ & An \\
\hline Vaccinium myrtillus & 2 & $\mathrm{Hu}$ & $\mathrm{Ch}$ & $\mathrm{R}$ & Zooch \\
\hline Verbascum lychnitis & 1 & Mx & $\mathrm{H}$ & $\mathrm{R}$ & An/Zooch \\
\hline Verbascum thapsus & 14 & Mx, D & $\mathrm{H}$ & $\mathrm{R}$ & An/Zooch \\
\hline Veronica chamaedrys & 1 & $\mathrm{Cc}, \mathrm{Hu}$ & $\mathrm{C}$ & $\mathrm{R}$ & Hy \\
\hline Vicia angustifolia & 1 & Mx & $\mathrm{T}$ & A & $\mathrm{Au}$ \\
\hline Vicia cracca & 71 & $\mathrm{Mx}, \mathrm{Hu}$ & $\mathrm{H}$ & $\mathrm{R}$ & $\mathrm{Au}$ \\
\hline Viola arvensis & 5 & $\mathrm{Mx}, \mathrm{D}, \mathrm{Hu}$ & $\mathrm{T}$ & A & Zooch \\
\hline Viola riviniana & 1 & $\mathrm{Hu}$ & $\mathrm{H}$ & $\mathrm{R}$ & Zooch \\
\hline
\end{tabular}


Woch M. W., RAdWAŃSKa M., STEFAnOwicz A. M.

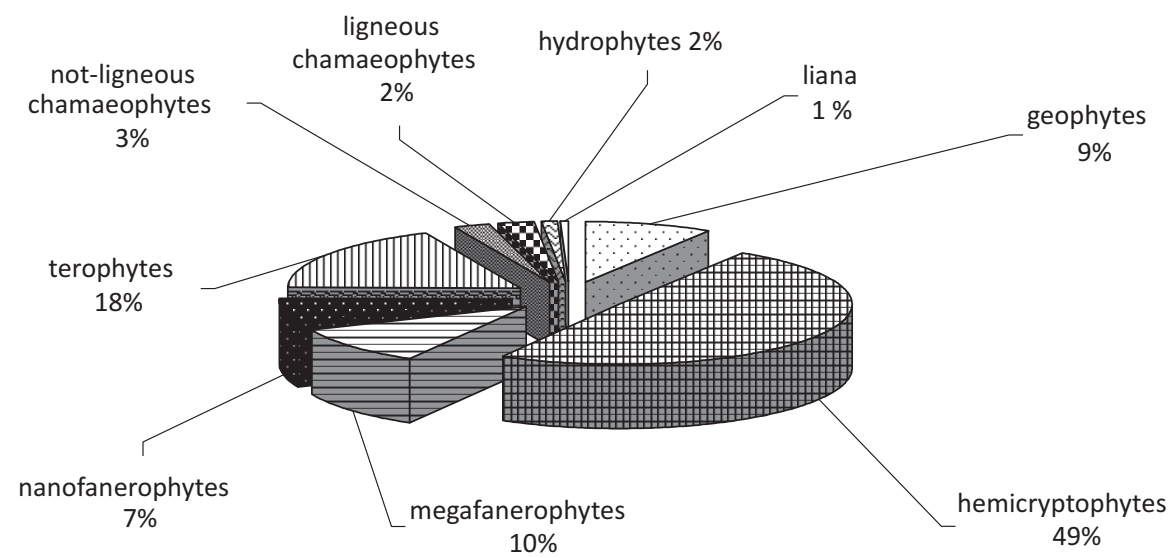

Fig. 4. Raunkiaer life forms in the flora (\%).

communities) (2\%), ephemerophytes (alien species that have been casually introduced into the territory) (3\%) and ergaziophytes (1\%) (cultivated plants, running wild) (Fig. 5). In the studied area the wind was the main vector of seed spreading (anemochory) (58\%), the second was animals (zoochory) (31\%). Other ways of dissemination were: autochory (6\%), anthropochory (4\%), hydrochory (3\%) and barochory (1 species) (Fig. 6). Among the noted

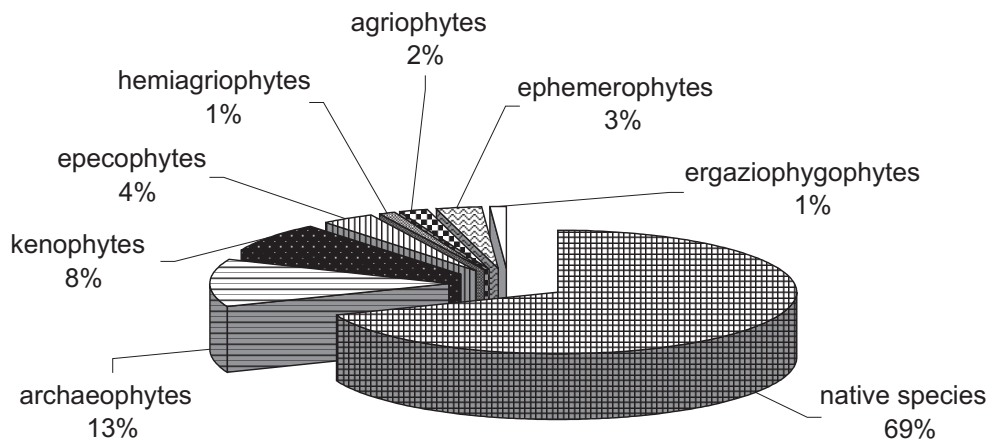

Fig. 5. Geographic-historical classification of the flora (\%).

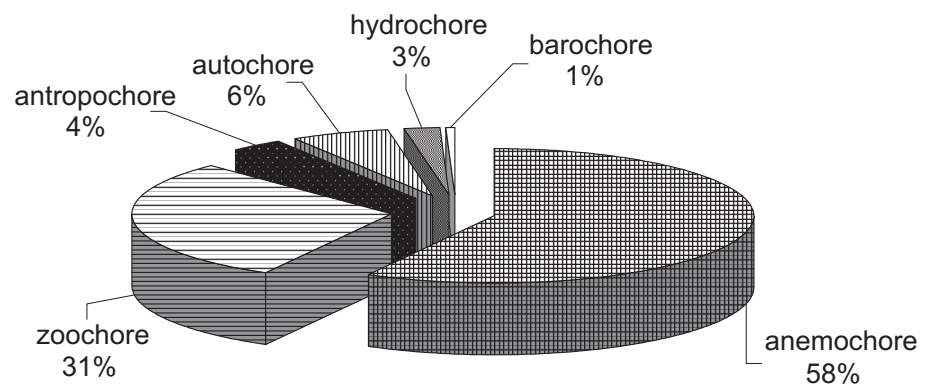

Fig. 6. Types of seed dispersion of the plant species (\%). 
species two - Epipactis atrorubens and E. helleborine - are strictly protected in Poland, and three are partly protected: Convallaria majalis, Frangula alnus and Ononis spinosa. Interesting was the presence of a few 2-3 m patches of a new expansive kenophyte of south-eastern origin - Typha laxmannii.

\section{Discussion}

The large variety of habitat conditions in these post-mining areas was influenced by the variation in substratum physicochemical parameters, such as humidity, exposure, texture, acidity, concentration of elements indispensable to plants and xenobiotics. The six discerned basic substratum types were characterized by distinct flora. The variability and instability of the substratum influences the diversity of the habitats, and various degrees of disintegration of the soil substrate may occur at different depths (ROSTAŃSKI 2006). Carbon shale with coal as well as culm substrata of sparsely overgrown areas in early succession stages contain more exchangeable magnesium in comparison to mixed or humus substrata. Carbon content, coarseness and compactness of the substratum were important factors determining plant diversity in the studied area. Plant species richness was highest on light calcareous and sandy soils with low content of clay fractions. In turn, it was lowest on heaps dominated by dust fraction with low content of calcium but a high content of coal, as in the case of culm and coal substrata. This relationship is corroborated by the results of CoHN et al. (2000) and WoźNIAK (2010). The high number of plant species on humus substratum may be connected with the advanced age (20-30 years) of succession in these places.

Generally, total $\mathrm{Fe}, \mathrm{Pb}, \mathrm{Zn}$ and $\mathrm{Tl}$ as well as exchangeable $\mathrm{Fe}, \mathrm{Mn}, \mathrm{Zn}$ and available $\mathrm{P}$ and water-soluble $\mathrm{Zn}$ content in soil increased with increasing share of coal wastes in a substratum. It is caused by high content of iron sulfides, mainly pyrite and traces of chalcopyrite, galena, marcasite, and sphalerite in coals and their associated sediments (LOTTERMOSER 2010). Thallium is often found accompanying these minerals. Total $\mathrm{Tl}$ concentrations in the studied soils varied from 5.98 to $14.59 \mathrm{mg} \mathrm{kg}^{-1}$. This indicates that $\mathrm{Tl}$ concentrations in soils significantly exceeded $\mathrm{Tl}$ concentrations found in unpolluted soils. Kabata-Pendias and Pendias (1993) stated that unpolluted soils worldwide contain from 0.02 to $2.8 \mathrm{mg} \mathrm{Tl} \mathrm{kg}^{-1}$, while in Poland they contain from 0.01 to $0.4 \mathrm{mg} \mathrm{Tl} \mathrm{kg}^{-1}$. This means that the most polluted substratum in our study contained ca 37 times more thallium than unpolluted soils in Poland. Thallium is an element rarely studied in Europe, to a much lesser degree than other toxic elements. High $\mathrm{Tl}$ concentrations in such sites may threaten the environment, because thallium is a highly toxic metal and it may be accumulated in plants and animals living at polluted sites (DMOWSKI and BADUREK 2002).

In the approximately 20 ha of the studied area of the former Siersza hard coal mine, 197 species of plants belonging to 53 families have been reported, which indicates a high species richness in this small area. The species composition of the spontaneously emerging plant cover on the dumps and wastelands is conditioned by the occurrence of a certain pool of species in its closest vicinity as well as some of them having an ecological amplitude allowing effective colonization of such places (BRÄNDLE et al. 2003). This is why the majority of the flora of the studied area was mostly constituted by species occurring within the surrounding $5 \mathrm{~km}$ (Holl 2002, SudER and CABAEA 2004, WoCH 2007). This is a typical regularity for various types of post-industrial places, where primary succession on the bare 
substratum will occur, such as quarries, sandpits, sedimentation tanks, dumps of various industrial materials (e.g. JochIMSEN 1996; CoHN et al. 2000; GRODZIŃsKA et al. 2001; RosTAŃSKI 2006; Woch 2007, 2012; WoźNIAK 2010).

The predominance of anemochoric plants indicates that wind dispersal is one of the most important features of plants that conquer the area first. Apart from the production of a large number of seeds, it is a basic feature of pioneer species expanding into a distant area, which direct their main effort towards migration (KREBS 2009). The role of zoochory increases considerably together with the time of the succession, playing an ever greater role in the later stages of plant cover formation. It is most probably connected with a parallel colonization of such areas by an ever greater number of animal species as well as an increase in their population. Native species dominated the flora of the studied areas (69\%), a share comparable to similar areas in Central Europe, where it is $70 \%$ on average (CABAtA and JARZĄBEK 1999, RostAŃSKi 2000, LACINA and KOUTECKÝ 2005).

Species alien to the flora of a certain area are more frequently encountered on newly anthropogenically affected areas, where they encounter weaker competition from native species. Hence a third of the studied flora was constituted by species alien to the Polish flora - anthropophytes $(31 \%)$. This group included new arrivals after the $15^{\text {th }}$ century (neophytes) (19\%), as well as arrivals from early historical times (archaeophytes) (12\%). Such a relatively high participation of neophytes is characteristic for young manmade structures with unstabilized plant cover. Species alien to Europe, such as Robinia pseudacacia and Solidago canadensis, were frequent and common in the research area, as well as the less frequent Acer negundo, Impatiens glandulifera, I. parviflora, Padus serotina and Quercus rubra. The abundance of these plants is characteristic for similar anthropogenic sites (CABAŁA and SYPIEŃ 1987, RosTAŃSKi 2006). The rapid diffusion of these species is result of their increased ecological flexibility in a new environment; especially anthropogenic soils. Northern American trees and shrubs are especially invasive, including Robinia pseudacacia, Quercus rubra and Padus serotina, which were introduced into Polish industrial areas in great numbers in the second half of the $20^{\text {th }}$ century. Robinia pseudacacia coexists with root nodule bacteria significantly increasing the content of nitrates in the substratum (SUDNIK-WÓJCIKOWSKA 2011). This has a positive effect on the soil formation process, but it can also hinder the appearance of native species and favor the succession of other alien species, which are mostly nitrophilous; similarly, diasporas of Quercus rubra and Padus serotina easily infiltrated the researched area zoochorically from the nearby forests. When it comes to plants, the formation of a stable mosaic of patches dominated by expansive native grasses (apophytes) such as Agrostis stolonifera, Calamagrostis epigejos, and invasive alien species as well as Solidago canadensis and S. gigantea within the first years of succession is a characteristic phenomenon. Such a superficially dominating vegetation type may in turn persist for many years. The presence of the new, expansive neophyte Typha laxmannii was interesting. In Poland, 29 localities are known, the first being discovered in 1988 near Kielce. In Europe T. laxmannii occurs naturally in Bulgaria, Romania, and the Ukraine, as well as the south-western part of Russia. In the south-western part of the range of occurrence it is a synanthropic species, noted in the Czech Republic, France, Germany, Slovakia, Slovenia as well as Italy (BARYLA et al. 2005). The next three found localities show that the species is spreading rapidly in anthropogenically modified habitats. T. laxmannii colonization may be connected with anemochoric penetration of the species from the abundant 
nearby populations occurring in a locality $3 \mathrm{~km}$ west of the researched area (WocH 2005). On the other hand, the discoveries of garden-grown ephemerophytes such as Antirrhinum majus, Calendula officinalis, Convallaria majalis, Fragaria x ananassa, or Solanum tuberosum are connected with the illegal storage of various types of waste.

Perennial species were predominant in the flora of the researched area $(82 \%, 49 \%$ of hemicryptophytes); the second group was constituted by annual plants (therophytes) (18\%). These values are comparable with the results of earlier studies performed in similar places, where in general the participation of hemicryptophytes is between 33 and $60 \%$, while the participation of therophytes is between 19 and 30\% (CABAEA and SYPEN 1987; TOKARSKA-GuZIK et al. 1991; CABAŁA and JARZĄEK 1999; WoRYNA and RostAŃSKI 2003; ROSTAÑSKI 1997, 2000, 2006). The large participation of therophytes in initial habitats and xeric areas is connected with the fact that completing a life cycle within an often short vegetation season is an important stress tolerance adaptation (MADON and MÉDAIL 1997). Habitats with coal ashes and carboniferous shale with coal substratum were only slightly overgrown with plants, the large participation of therophytes in it signifies that it is in early succession stages. On a mixed substratum, the largest number of xerothermic species was reported; this is connected with easy water permeability, quick heating, as well as an increased content of calcium carbonate. Ononis spinosa grew on it - a xerothermic grassland species protected in Poland. In some of the waste dumps with the mostly advanced stages of succession, communities similar to mixed forests have been formed, in which the layer of trees included Betula pendula, Pinus sylvestris, Populus tremula and Salix caprea, and undergrowth connected with Calluna vulgaris, Deschampsia flexuosa, Poa nemoralis and Vaccinium myrtillus forest species. Compact turf was formed and a 5-10 cm layer of topsoil was accumulated (humus substratum). Greater shading in this part of the area, the humidity of the northern slopes, as well as the deposition of organic matter from the nearby forest was responsible for rapid forestation. The location on the outskirts of the former mining plant as well the age of the oldest trees estimated on the basis of the number of branch whorls to be between 20 and 30 years implies that the succession in these places already started when the mine was operating. In the humus substratum only, in the favorable climate under the trees there were forest species strictly protected by the Polish law: Epipactis atrorubens, E. helleborine as well as the partially protected Frangula alnus. Plant patches near the forest with rare species of orchids occurred also on the most advanced succession stages in post-mining dumps in the Upper Silesia Coalfield (CABAŁa and SyPieŃ 1987, CABAŁA and JARZĄBEK 1999, RostAŃSKi 2006), Lower Silesia Coalfield (KUCZYŃSKAet al. 1984), as well as in post-mining dumps in England and Germany (AsH et al. 1994, EsFelD et al. 2008).

The studies carried out in various post-mining plants reveal their high species abundance as well the occurrence there of rare protected plants (WOŹNIAK and ROSTAŃSKI 2001, RostaŃSKI and MichALSKA 2003, RosTAŃSKi 2006). They can be very important in the protection of biodiversity on the condition that their habitat variety is preserved, leaving them without carrying out recultivation (LuNDHOLM and RICHARDSON 2010). Post-mining areas such as waste dumps remaining after the Siersza hard coal mine should be protected in various ways and used as research and education facilities, where long-term ecological research would be performed, and learning trails would popularize issues of plant cover development in an untypical manmade habitat. It also constitutes the industrial heritage of the Kraków coalfield. 


\section{References}

Ash, H. J., Gemmell, R. P., Bradshaw, A. D., 1994: The introduction of native plant species on industrial waste heaps: a test of immigration and other factors affecting primary succession. Journal of Applied Ecology 31, 74-84.

BAIG, M. N., 1992: Natural revegetation of coal mine spoils in the Rocky Mountains of Alberta and its significance for species selection in land restoration. Mountain Research and Development 12, 285-300.

Baryla, J., Bróż, E., Czylok, A., Michalewska, A., Nikiel, A., Nobis, M., Piwowarczyk, R., PoloczeK, A., 2005: Typha laxmannii Lepech. The new, expansive kenophyte in Poland: distribution and taxonomy. Acta Societatis Botanicorum Poloniae 74, 25-28.

BrÄNDle, M., DurKa, W., Krug, H., BrandL, R., 2003: The assembly of local communities: Plants and birds in non-reclaimed mining sites. Ecography 26, 652-660.

CABAlA, S., JARZĄBeK, Z., 1999: The flora and vegetation of postindustrial dumps in Chorzów, 1. Analysis of the flora. Archiwum Ochrony Środowiska 25, 133-153.

CABAŁA, S., SyPień, B., 1987: Development of vegetation on the coal mine spoil heaps, of the Upper Silesian Industrial Region (in Polish). Archiwum Ochrony Środowiska 3-4, $169-184$.

Cohn E., V. J., Rostański, A., Tokarskka-Guzik, B., Trueman, I. C., Woźniak, G., 2000: The flora and vegetation of an old Solvay process tip in Jaworzno (Upper Silesia, Poland). Acta Societatis Botanicorum Poloniae 70, 47-60.

DMOWski, K., BADUREK, M., 2002: Thallium contamination of selected plants and fungi in the vicinity of the Boleslaw zinc smelter in Bukowno (S. Poland). Preliminary study. Acta Biologica Cracoviensia series Botanica 44, 57-61.

Esfeld, K., Hensen, I., Wesche, K., Jakob, S., Tischew, S., Blattner, F., 2008: Molecular data indicate multiple independent colonizations of former lignite mining areas in Eastern Germany by Epipactis palustris (Orchidaceae). Biodiversity and Conservation 17, 2441-2453.

GiBson, D. J., Johnson, F. L., RIsser, P. G., 1985: Revegetation of unreckaimed coal strip mines in Oklahoma. II. Plant communities. Reclamation and Revegetation Research 4, $31-47$.

Grodzińska, K., Korzeniak, U., Szarek-Lukaszewska, G., GodziK, B., 2001: Colonization of zinc mine spoils in southern Poland - preliminary studies on vegetation, seed rain and seed bank. Fragmenta Floristica et Geobotanica Polonica 45, 123-145.

HoLL, K. D., 2002: Long-term vegetation recovery on reclaimed coal surface mines in the eastern USA. Journal of Applied Ecology 39, 960-970.

Holl, K. D., Cairns, J. J., 2002: Monitoring and appraisal. In: Perrow, M. R., DAVY., A. J. (eds), Handbook of Ecological Restoration, 411-432. Cambridge University Press, Cambridge.

INTERNATIONAL ORGANIZATION FOR STANDARDIZATION, 1994: International standard of soil quality - Determination of pH (ISO 10390).

INTERNATIONAL ORGANIZATION FOR STANDARDIZATION, 1995a: International standard of soil quality - Determination of organic and total carbon after dry combustion (elementary analysis)(ISO 10694). 
INTERNATIONAL ORGANIZATION FOR STANDARDIZATION, 1995b: International standard of soil quality - Determination of total nitrogen - Modified Kjeldahl method (ISO 11261).

INTERNATIONAL ORGANIZATION FOR STANDARDIZATION, 1995c: International standard of soil quality - Determination of the potential cation exchange capacity and exchangeable cations using barium chloride solution buffered at $\mathrm{pH}=8.1$ (ISO 13536).

INTERNATIONAL ORGANIZATION FOR STANDARDIZATION, 1998: International standard of soil quality - Determination of particle size distribution in mineral soil material - Method by sieving and sedimentation (ISO 11277).

Jochimsen, M. E. A., 1996: Reclamation of colliery mine spoil founded on natural succession. Water, Air, and Soil Pollution 91, 99-108.

Kabata-Pendias, A., Pendias, H., 1993: Biogeochemistry of trace elements (in Polish). PWN, Warszawa.

KIRYK, F. (ed.)., 1994: Trzebinia. A draft of town and region history (in Polish). Secesja, Kraków.

KondRACKI, J., 2000: Regional geography of Poland (in Polish). PWN, Warszawa.

KreBS, C. J., 2009: Ecology: The Experimental Analysis of Distribution and Abundance. Benjamin Cummings, San Francisco.

Kuczyńska, I., Pender, K., RyszKa-Jarosz, A., 1984: The vegetation of selected waste-heaps of the coalmine »Victoria « in Walbrzych. Acta Universitatis Wratislaviensis, Prace Botaniczne 27, 35-60.

LACINA, L., KoUTECKÝ, T., 2005: Biogeographical and geobiocoenological aspects of deep coal mining and its impacts on nature and landscape in the Ostrava Region. Moravian Geographical Reports 13, 34-48.

LotTermoser, B. G., 2010: Mine Wastes: Characterization, Treatment and Environmental Impacts. Springer Heidelberg Dordrecht, London, New York.

LuKen, J. O., 1990: Directing Ecological Succession. Chapman and Hall, London.

Lundholm, J. T., RichaRdson, P. J., 2010: Habitat analogues for reconciliation ecology in urban and industrial environments. Journal of Applied Ecology 47, 966-975.

Madon, O., MédAIL, F., 1997: The ecological significance of annuals on a Mediterranean grassland (Mt Ventoux, France). Plant Ecology 129, 189-199.

MAKINECI, E., GunGOR, B. S., KuMBASLI, M., 2011: Natural plant revegetation on reclaimed coal mine landscapes in Agacli-Istanbul. African Journal of Biotechnology 10, 32483259 .

Olsen, S. R., Cole, C. V., Watanabe, F. S., Dean, L. A., 1954: Estimation of available phosphorus in soils by extraction with sodium bicarbonate. U. S. Department of Agriculture. Circular 939. Governmental Printing Office Washington D. C.

Pickett, S. T. A., Collins, S. L., Armesto, J. J., 1987: Models, mechanisms and pathways of succession. Botanical Review 53, 335-371.

PodbielKowski, Z., 1995: Migrations of plants (in Polish). WSiP, Warszawa.

RostaŃSKI, A., 1997: Spontaneous flora on coal spoil heaps in Upper Silesia (Poland). Archiwum Ochrony Środowiska 23, 159-165. 
RostAŃSKI, A., 2000: Spontaneous flora of post-industrial sites of the Upper Silesia region. A summary of investigations (1989-1999) (in Polish). Acta Biologica Silesiana 35, 131-154.

RostAŃSKI, A., 2006: Spontaneus plant cover on colliery spoil heaps in Upper Silesia (Southern Poland) (in Polish). Wydawnictwa Uniwersytetu Śląskiego, Katowice.

Rostański, A., MichalskA, M., 2003: Rich population of orchid (Epipactis palustris (1.) Cranz) on a zinc-colliery heap in Świętochlowice-Chiropaczów (Upper Silesia, Poland) (in Polish). Archiwum Ochrony Środowiska 29, 115-118.

RUTKOwsKi, L., 2004: A key to identification of vascular plants of lowland Poland (in Polish). PWN, Warszawa.

Suder, A., CabalA, S., 2004: Vegetation of Trzebinia town in conditions of strong human impact, 1, Vascular flora. Archiwum Ochrony Środowiska 30, 77-104.

SudNiK-WóJcikowska, B., 2011: Synanthropic plants (in Polish). Multico, Warszawa.

TOKARSKA-GuZIK, B., RostAŃSKI, A., KLOTZ, S., 1991: Vegetation of zinc smelter spoil heap in Katowice-Welnowiec (in Polish). Acta Biologica Silesiana 19, 94-102.

Witkowska-Kita, B., Baic, I., Biel, K., Ostatkiewicz, A., 2010, The program of environmental protection for the Trzebinia district on years 2010-2013 (in Polish), Official Website of the Trzebinia Town Council. Retrieved December 7, 2012, from http: www.trzebinia.pl

Woch, M. W., 2005: Spreading of Typha laxmannii (Typhaceae) in the Silesian Upland (in Polish). Fragmenta Floristica et Geobotanica Polonica 12, 176-179.

Woch, M. W., 2007: Flora and vegetation of the drift in the Szczakowa Sand Pit S.A. (in Polish). Fragmenta Floristica et Geobotanica Polonica 14, 281-309.

Woch, M. W., 2012: New and rare synanthropic plant species of the Polish flora on closed sedimentation ponds of the Siersza Power Plant (in Polish). Fragmenta Floristica et Geobotanica Polonica 19, 29-38.

Woryna, G., RostaŃSKi, A., 2003: Vascular flora of coal-mine spoil heaps in Ruda Śląska (Upper Silesia, Poland) (in Polish). Archiwum Ochrony Środowiska 29, 77-91.

WoźNIAK, G., 1998: Primary succession on the sedimentation pools of coal mine. Phytocoenosis 10, 189-197.

WoźNIAK, G., RostańsKi, A., 2001: Chamaenerion palustre Scop. as a frequent apophyte in plant communities of post-industrial waste sites. Natura Silesiae Superioris Suplement $5,55-66$.

WoźNIAK, G., 2010: Diversity of vegetation on coal-mine heaps of the Upper Silesia (Poland) (in Polish). Wydawnictwo Instytutu Botaniki PAN, Kraków. 